\title{
A Genetic Improved Parametric Clustering to Optimize WSN Communication
}

\author{
Himani Kathuria \\ Student M.Tech \\ Panipat college of Eng and technology
}

\author{
Sachin Dhawan \\ Associate Member AIET Assistant Professor \\ Panipat institute of Eng and technology
}

\begin{abstract}
Sensor network is critical real time network used in application specific areas. The restricted energy and coverage increases the communication criticality. Because of this, the network follows an architecture driven communication for effective resource utilization. In this paper, a mobility adaptive cluster optimization model is presented to improve the network communication. At the earlier phase of this model, the individual node analysis is applied under load, stability, energy and connectivity parameters. Based on which the cluster election is performed. After identifying the clusters, the range driven separation is performed to provide the single or multihop path. In the final stage, the route optimization is provided for external cluster nodes as well as aggregative cluster nodes. For route generation, a genetic driven evolutionary process is defined. The fitness rule for genetic is applied under stability, distance and energy parameters. Finally, all the cluster heads will deliver the aggregative data to base station. The simulation results show that the model has improved the network life and communication.
\end{abstract}

\section{Keywords}

WSN, Fitness rule of Genetic algorithm.

\section{INTRODUCTION}

Wireless Sensor Network have grown enormously and become attractive in wide variety of application because of their low cost, low power, self organizing behavior in harsh environment and small size. Routing plays main role in wireless sensor network. There are many routing protocols like: location based, multipath, data centric, mobility based etc [2].

Wireless Sensor Network (WSN) is composed by a large amount of low-cost micro sensor node distributed in monitored area. It is spatially distributed autonomous sensors to monitor physical or environmental condition such as temperature, sound, pressure, etc. and to cooperatively pass their data through the network to a main location. The networks are bi-directional, also enabling control of sensor activity. The development of wireless sensor networks was motivated by military applications such as battlefield surveillance; today such networks are used in many industrial and consumer applications, such as industrial process monitoring and control, machine health monitoring, and so on. [7].

Wireless sensor network consist of hundred to thousand of nodes that are dispersed in sensing region to sense the environment and sensed data is than send to the sink. A sensor node is made of four components a sensing unit, a processing unit, a communication unit, a power unit. A Sensing unit consists of no of sensors and analog to digital convertor. The sensors sense the data which is a physical phenomenon and generate a analog signal. The analog to digital converter convert that analog signal to digital signal and send that data to processing unit .The processing unit has limited energy storage and a processor that controls all sensors. A communication unit use radio signals for data transmission between nodes. The most important unit of sensor nodes is power unit which supply power to the nodes .we can have more components in sensor node depending upon the application. [2]

In wireless sensor network wireless medium is used for communication between each other and nodes can be homogeneous and heterogenous.In Homogenous network all the nodes are given with same amount of energy and in Heterogeneous some nodes are equipped with higher energy as compare to other nodes.[2]

To keep the cost of entire sensor network low, cost of each sensor needs to be reduced. It is also important to use tiny sensor nodes. A smaller size makes it easier for a sensor to be embedded in the environment in which it wants to. WSNs may also have a lot of redundant data since multiple sensors can sense similar information. The sensed data therefore needs to be aggregated to decrease the number of transmissions in the network, reducing bandwidth usage and eliminating unnecessary energy consumption in both transmission and reception [2].

Clustering is major technique that has been defined to minimize this energy dissipation while trans-mission there is one $\mathrm{CH}$ and associated member nodes in one cluster. As in wireless sensor network our main objective is to increase the lifetime of network and reduction of energy consumption so clustering is one of the most popular routing method that was described no of authors. When the sensor nodes are grouped for local aggregation it is called clustering. When sink or base station is often located far from the network in that case instead of using direct communication clustering is much better to save the energy. All the sensor nodes are grouped into the clusters and each cluster has its on cluster head that will directly communicate with sink. The cluster head is being chosen by cluster member or sink. Data aggregation at cluster head will reduce the number of transmissions to the sink and improves energy efficiency and lifetime of the network.

Clustering is used to balance the load in the network. Clustering is an example of layered protocols where the network consists of several clusters of sensor nodes. Each cluster head will take data from its cluster member and aggregated data is being sent to base station. The transmission between cluster head and cluster member is called intra cluster communication and the transmission between cluster head and sink is called inter cluster communication. Clustering reduce routing overhead and make network more 
stable [2].

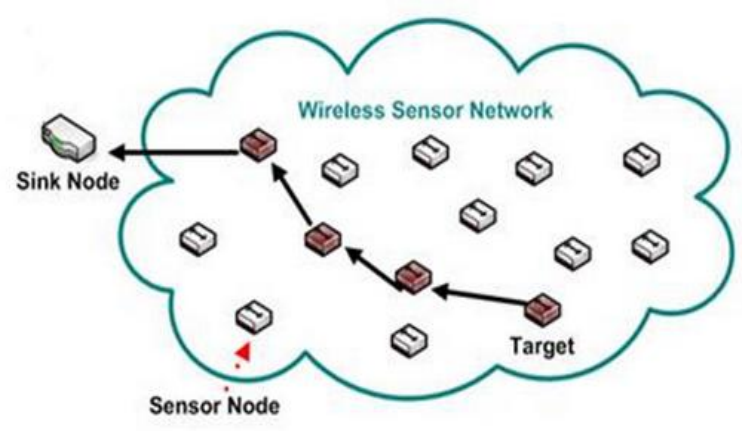

Figure 1 structure of wireless sensor network

As we know that the lifetime of network and how much data they can collect entirely depends on the limited amount of battery capabilities. Many Researchers' has addressed this situation form different aspects and defined different type of energy efficient routing protocol.

LEACH stands for Low Energy Adaptive Clustering Hierarchy. It is the one of the most popular clustering algorithm that was used in wireless sensor network. In this network is divided into clusters based on the signal strength of all the sensors. It is one of the most popular hierarchal routing protocol in sensor network. LEACH form all the network into cluster and then elect one cluster head for each cluster formed. All the sensed data by each sensor nodes will be transmitted to cluster head of each cluster and then each cluster head acts as router to really all data to sink. It is one of the most popular distributed cluster based algorithm

LEACH uses time division multiple access (TDMA) principal to avoid collision. In LEACH each nodes based on probabilistic behavior becomes cluster head to maintain balance energy consumption in the network. In this technique the cluster head is selected without considering the residual energy and other properties of sensor nodes. The random through which cluster head is being elected does not guarantee the distribution of clusters in the network. The drawback of this protocol is that it ignores the inter cluster communication and assumes that each node can directly communicate with sink node which is not possible as each not has limitation due to communication range restriction.

The author protocol that is being used is PEGASIS .This technique is introduced to overcome the limitation of LEACH.PEGASIS form chain of sensor node so each node need to communicate only with it is neighbored node so it make this technique more efficient. In this one the chain of the nodes is selected to send the sensed data from sensor node to sink.

After that a new protocol is proposed that was HEED. It stands for Hybrid energy efficient distributed clustering. It is also distributed energy efficient clustering protocol that is extension of LEACH. In this technique cluster head selection is based on hybrid of the node residual energy and a secondary parameter such as node proximity to its neighbors or node degree. The cluster formation is achieved by iterative approach. HEED operates in multihop networks using an adaptive transmission powers in interclustering communication. HEED can evenly distribute the cluster head in the sensing area by local competition.
In both LEACH and HEED, they form cluster by considering only one node which will die much faster than other node is the cluster this will create hotspot problem in which node close to cluster head will die earlier than other node and network lifetime will decrease

Other techniques are LEACH-C .It stands for centralized low energy adaptive clustering hierarchy. As in LEACH where each node self configure them self into cluster in LEACH-C base station is used for cluster formation. Initially each node sends its information (Energy and location) to the base station and base station use this information to predetermine the cluster head and the clusters in the network. The cluster as chosen in such a manner that each sensor node require minimum energy to transmit the data the to its cluster head.

There is one more technique that is proposed TL-LEACH. It stands for two levels LEACH and it is the enhanced version of LEACH. In this Technique same method is used for cluster head election and cluster formation. In the algorithm two sensor nodes are elected as cluster head, one is known as primary cluster head and other is called as secondary cluster head .Both the cluster head can communicate with each other but secondary node can communicate with other node in the cluster. In this cluster tree is formed either top down approach or bottom up approach.

We also study about SEP. It stands for stable election protocol. SEP is proposed for heterogeneous aware networks .It Is mainly used to prolong the time interval before the first node dies which is also refer as stability period of network which is crucial for many where the feedback from many sensor network is SEP is based on weighted election probabilities of each node to become cluster head according to the remaining energy in each node. SEP always prolongs the stability period compared to (and that the average throughput is greater than) the one obtained using current clustering protocols. SEP always prolongs the stability period compared to (and that the average throughput is greater than) the one obtained using current clustering protocols. SEP is more resilient than LEACH in judiciously consuming the extra energy of advanced nodes - SEP yields longer stability region for higher values of extra energy.

In this paper we are using two phase parameter specific optimization model is presented for mobility driven sensor network. In the first phase, load, stability, connectivity, energy and probability vector based analysis vector is defined for cluster head election. After forming the clusters, the range division is applied to select the internal and external nodes. Thus routing is applied based on stability, distance and energy based analysis. As the data is collected on cluster heads, the final aggregate communication route is established using genetic based route formation model. The fitness rule for genetic is applied under stability, distance and energy parameters. Finally, all the cluster heads will deliver the aggregative data to base station.

The rest of the paper is organized as in Section II Research Methodology is defined, in Section III Simulation results are shown and in Section IV and V conclusion and future work is defined

\section{RESEARCH METHODOLOGY}

In this work, an improved clustering architecture is defined for mobile sensor network. A mobile sensor network is the most critical form of sensor network in which energy nodes are defined with mobility. At the early stage, the node stability and load analysis are evaluated with energy and probability 
vectors for cluster head selection. Once the clustering is done, the next work is to divide the network in zones so that the internal and external nodes are identified. The internal nodes will perform the direct communication with cluster head, whereas the external nodes will perform multi-hop communication. The multi-hop route formation will be done here under energy, distance and stability vectors. In the final stage, the aggregative route will be established between the cluster heads. This route formation will be done using genetic approach. The work is about to provide effective communication in clustered network.

The work is mainly divided in two main stages. In first phase, a stability and load optimized clustering is performed. The balanced clustered model has reduced the switching in the cluster formation. While performing the clustering, the identification of optimal energy node is done based on the defined parameter. The parameters considered for cluster formation includes

- $\quad$ Node Energy

- Coverage

- $\quad$ Stability

- Load

- $\quad$ Selection Probability

These parameters are applied while identifying the cluster head in a nested form. At first the probabilistic analysis and the node energy are analyzed to check for minimum eligibility of a node. Later on the stability and coverage based load parameters are applied for most

Effective node identification. After setting up the effective node, the cluster region is defined.

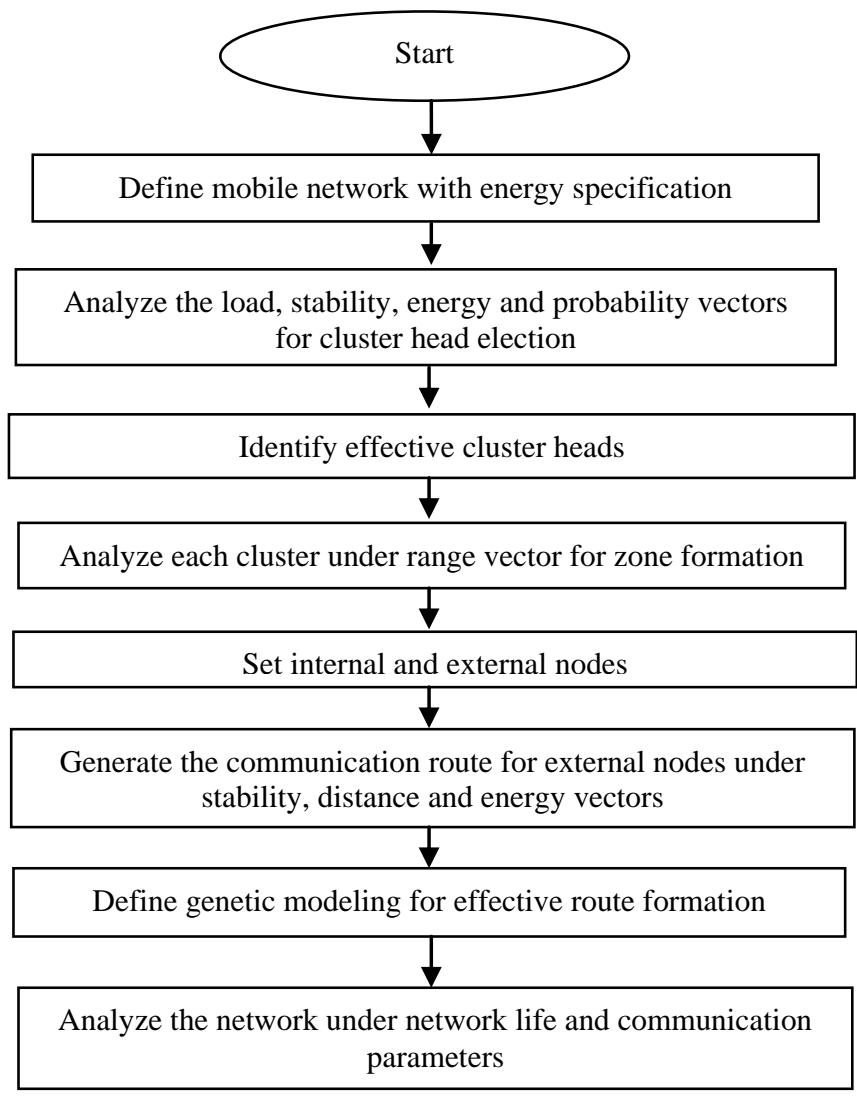

Figure 2 Flow Chart

\subsection{Algorithm Genetics}

The work is here defined using genetic approach to provide the effective route formulation using genetic approach. The nodes are here defined as the random energy nodes and with specification of cluster heads. The genetic process used the cluster nodes are the raw population set and defined the population formation. The random sequence set is defined between the node and cluster head specification. As the multiple paths are generated, it formed the overall population set. This population is then processed under genetic approach with series of operators to generate the communication path.

The Genetics algorithm is the part of Evolutionary computing, a rapidly growing area. GA's was inspired by Darwin's theory about evolution -"Survival of the fittest". Also we can say that Genetic Algorithm is related to fittest individuals dominate over the weaker one's.

GA is a way of solving problems by mimicking process which uses' below:-

1. Selection

2. Crosses over

3. Mutation

4. Accepting

1. Selection:-

It is the first part of genetics algorithm, it is also called as reproduction. It is the like the first operator applied on population from selection or reproduction the chromosomes are selected to be parents to be crossover and produce offspring. In the best possible path is selected based on random encoding method. After that the fitness rule is applied based on minimum distance and minimum energy constraints.

\section{Crossover:-}

It is the second part, it is an operator that combines two chromosomes(parents) to produce new chromosomes (offspring). The idea behind the concept is that may be possible the offspring consist of the best possible characteristics of the parents. In the crossover from both the parents possible path best possible child is selected .The crossover is one point crossover, two point crossover, Uniform crossover or arithmetic crossover.

\section{Mutation:-}

After a crossover is performed after that mutation is performed, It is genetic operator used to maintain genetic diversity from one generation of chromosomes to other .Mutation alters one or more gene value in the chromosomes from its initial state which led to have totally new chromosomes similarly it is followed for paths also. The Mutation operators are flip bit, Boundary etc.

\section{Accepting:}

It is last step, it involves where we select our best optimized result.

The algorithmic specification for path generation using genetic approach is given here under

1. Generate the path sequence pool based on random encoding method

2. Define the fitness rule based on minimum distance and minimum energy constraints 
3. For $\mathrm{i}=1$ to MaxIterations

[Repeat steps 4 to 7 ]

4. \{

5. Parent $1=$ Selection(Population, Fitness)

Parent $2=$ Selection(Population, Fitness)

[Generate two parents using fitness rule specification

6. Child=ApplyDPXCrossover(Parent1,Parent2)

7. Path=Muratio(Child)

8. Recombine the obtained value in the population set.

9. \}

10. Return Optimized Sequence

11. Generate the graph of path sequence.

\section{SIMULATION RESULTS}

\section{Network Scenario}

A random placement based sensor network is defined to present the work. The scenario parameters considered in this work are listed here

\begin{tabular}{|c|c|}
\hline Parameters & $\underline{\text { Values }}$ \\
\hline Area & $100 \times 100$ \\
\hline Number of Nodes & 100 \\
\hline Initial Energy & 0 to $1 \mathrm{~J}$ \\
\hline Transmission Loss & $5 \mathrm{~nJ}$ \\
\hline Receiving Loss & $5 \mathrm{~nJ}$ \\
\hline Sensing Range & $30 \mathrm{mtr}$ \\
\hline $\begin{array}{c}\text { Load Threshold } \\
\text { Number of Communication } \\
\text { Rounds }\end{array}$ & 10 \\
\hline
\end{tabular}

Figure 3: Network Parameter

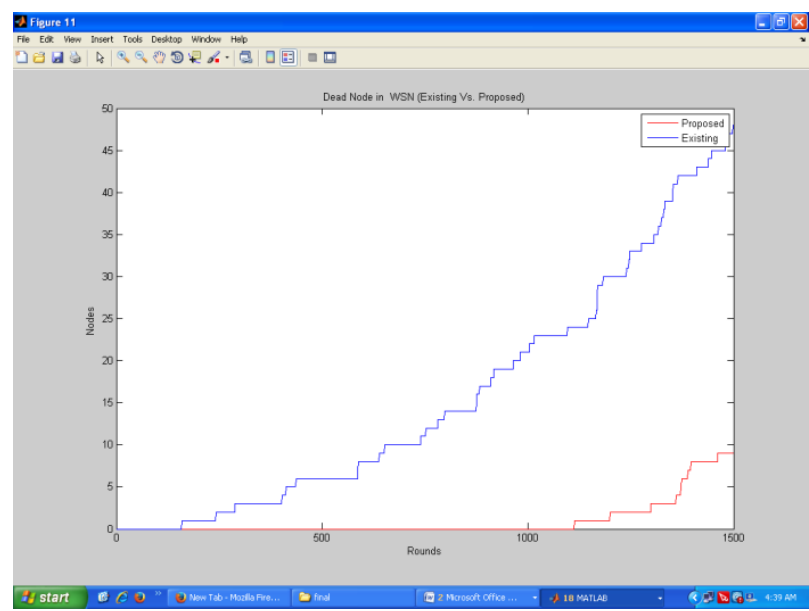

Figure 4 : Dead Node Analysis (Comparative)

Here figure 1 is showing the comparative analysis of existing and proposed work in terms of dead nodes. Here $\mathrm{x}$ axis represents the number of communication rounds and $y$ axis shows the dead nodes. Here figure shows that the numbers of dead nodes in existing work are 48 whereas in this proposed work only 9 nodes are dead.

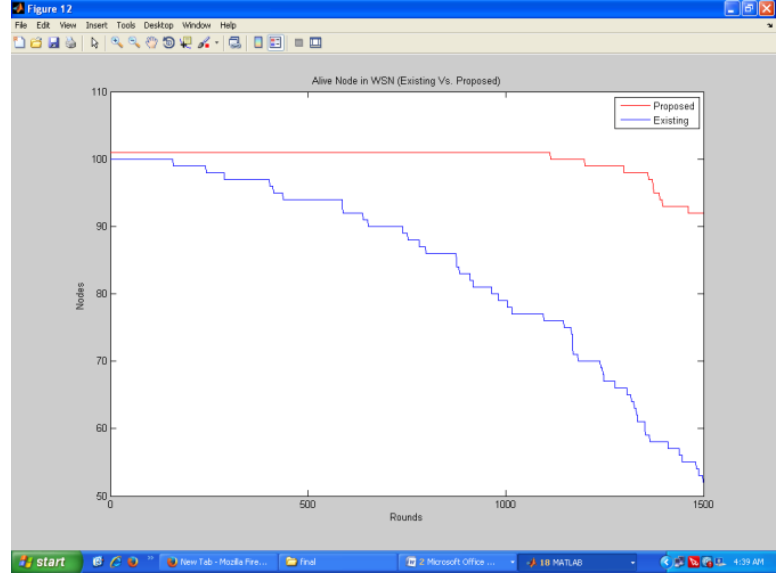

Figure 5 : Alive Node Analysis (Comparative)

Here figure 2 is showing the comparative analysis of existing and proposed work in terms of alive nodes. Here $\mathrm{x}$ axis represents the number of communication rounds and $y$ axis shows the alive nodes. Here figure shows that the numbers of alive nodes in existing work are 52 whereas in this proposed work only 91 nodes are alive.

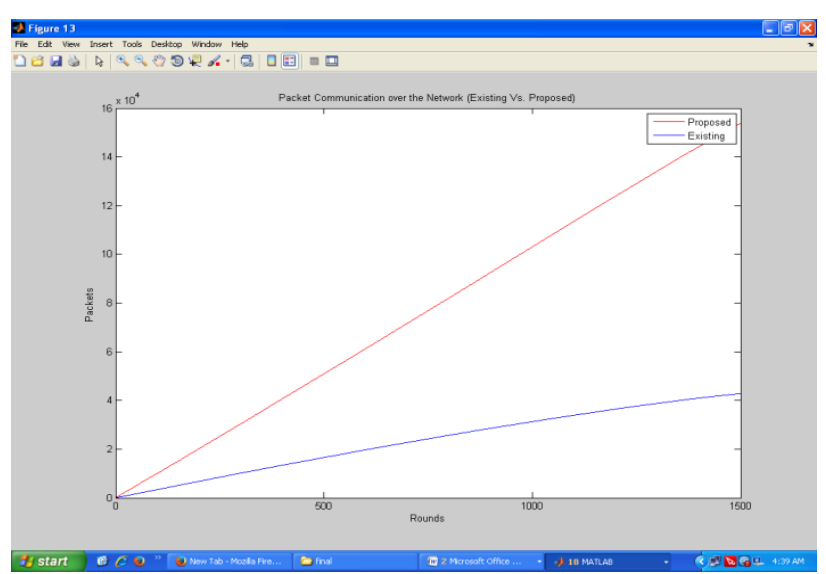

Figure 6 : Network Communication Analysis (Comparative)

Here figure 3 is showing the comparative analysis of existing and proposed work in terms of packet communication. Here $\mathrm{x}$ axis represents the number of communication rounds and $y$ axis shows the packet communication. Here figure shows that the packet communication in this proposed work is improved.

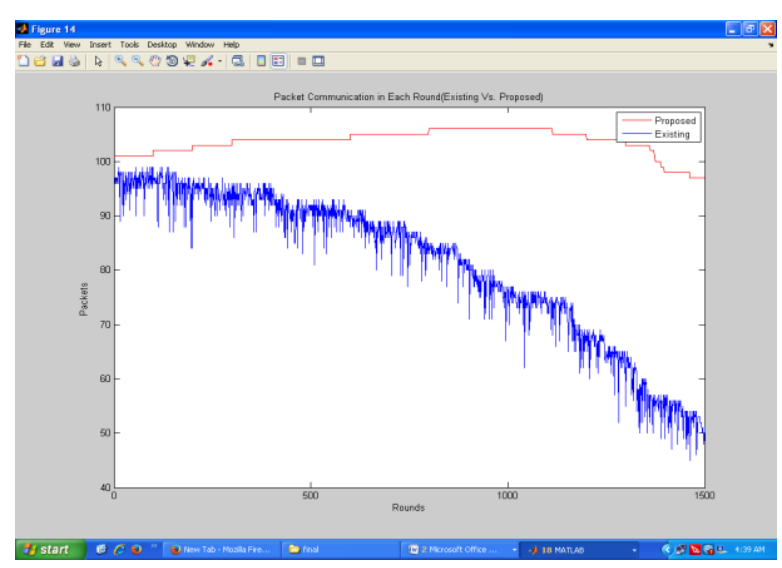

Figure 7 Round Based Network Communication Analysis (Comparative) 
Here figure 4 is showing the comparative analysis of existing and proposed work in terms of packet communication. Here $\mathrm{x}$ axis represents the number of communication rounds and $y$ axis shows the packet communication. Here figure shows that the packet communication in this proposed work is improved.

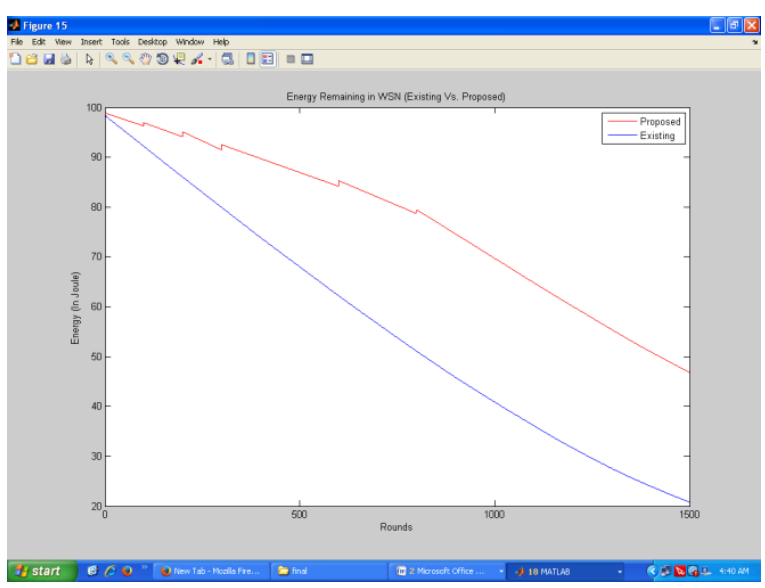

Figure 8 : Network Energy (Comparative)

Here figure 5 is showing the comparative analysis of existing and proposed work in terms of network energy. Here $\mathrm{x}$ axis represents the number of communication rounds and $y$ axis shows the packet energy. Here figure shows that the packet energy loss in this proposed work is is decreased.

\section{CONCLUSION}

In this paper, a dual phase architecture level optimization is provided. At the early phase, the cluster optimization is achieved by considering the load, connectivity and the energy parameters. Later on the route optimization is achieved for external cluster nodes as well as for the aggregative cluster path. The genetic driven method is applied under stability and load parameters to generate effective communication path. The simulation results show that the method has improved the communication and network life. In future, the encoded and secure communication can be applied against the fault. The encoded and compressed communication can improve the reliability and efficiency of work.

\section{REFERENCES}

[1] LI Han, "A Multiple-Hop Energy Efficient Clustered Algorithm for Heterogeneous WSN", Fourth International Conference on Multimedia Information Networking and Security, pp 183-186, 2012

[2] Naveen Sharma and Anand Navyar " A comprehensive Review of Cluster Based Energy Efficient Routing Protocols for Wireless Sensor network “, International Journal of Application or Innovation in Engg \& Management ISSN 2319-4847.In year 2010, Amir Mollanejad , Leili Mohammad Khanli , Mohammad Zeynali,Mohammad Zeynali

[3] Huan Chen, Cheng-Shong Wu, Yuan-Sun Chu, ChihChuan Cheng and Li-Kuang Tsai "Energy Residue Aware (ERA) Clustering Algorithm for Leach-based WirelessSensor Networks", Second International Conference on Systems and Networks Comm.

[4] Xia Li, Wang Gang, Liu Zongqi, Zhang Yanyan "An energy-efficient routing protocol based on particle swarm clustering algorithm and inter-cluster routing algorithm for WSN , 2013 IEEE
[5] Riham S. Elhabyan, and Mustapha C.E. Yagoub, Senior Member, IEEE "WEIGHTED TREE BASED ROUTING AND CLUSTERING PROTOCOL FOR WSN" , 2013 26th IEEE Canadian Conference Of Electrical And Computer Engineering (CCECE).

[6] Amir Mollanejad , Leili Mohammad Khanli , Mohammad Zeynali , Hadi Bahrbegi "EHRP: Novel Energy-aware Hierarchical Routing Protocol in Wireless Sensor Network", 2010 International Congress on Ultra Modern Telecommunications and Control Systems and Workshops (ICUMT).

[7] Tifenn Rault, Abdelmadjid Bouabdallah, Yacine Challal ,"WSN Lifetime Optimization through Controlled Sink Mobility and Packet Buffering, 2013 IEEE.

[8] Kee-Young Shin, Junkeun Song, JinWon Kim, Misun Yu, and Pyeong Soo Mah," REAR: Reliable Energy Aware Routing Protocol for Wireless Sensor Networks , 2007 ICACT2007.

[9] Ashanie Gunathillake and Kithsiri Samarasinghe ," An Unequal Clustering Algorithm for an Emergency Response Wireless Sensor Network", 2013 IEEE 9th International Conference on Mobile Ad-hoc and Sensor Networks.

[10] L. Malathi M.K. Chandrasekaran R.K. Gnanamurthy ," A NOVEL ROUTING PROTOCOL WITH LIFETIME MAXIMIZING CLUSTERING ALGORITHM FOR WSN", (C2012 IEEE.

[11] Akash Punhani and Nitin "A Qos Based Routing Using Genetic Algorithm”, 2011 IEEE

[12] Jisul Choe, Keecheon Kim ," EADD: Energy Aware Directed Diffusion for Wireless Sensor Networks", 2008 International Symposium on Parallel and Distributed Processing with Applications.

[13] Geoff V. Merrett, Neil M. White, Nick R. Harris and Bashir M. Al-Hashimi ," Energy-Aware Simulation for Wireless Sensor Networks", This full text paper was peer reviewed at the direction of IEEE Communications Society subject matter experts for publication in the IEEE Secon 2009 proceedings.

[14] Alphan Ulusoy, Member, IEEE, Ozgur Gurbuz, Member, IEEE, and Ahmet Onat, Member, IEEE , "Wireless Model-Based Predictive Networked Control System Over Cooperative Wireless Network", IEEE TRANSACTIONS ON INDUSTRIAL INFORMATICS, VOL. 7, NO. 1, FEBRUARY 2011.

[15] Mohamed K. Watfa, Haitham AlHassanieh, and Samir Selman ," Multi-Hop Wireless Energy Transfer in WSNs", IEEE COMMUNICATIONS LETTERS, VOL. 15, NO. 12, DECEMBER 2011

[16] S. Manfredi ," Reliable and energy-efficient cooperative routing algorithm for wireless monitoring systems", Published in IET Wireless Sensor Systems Received on 14th March 2011.

[17] Walid Bechkit , Mouloud Koudil , Yacine Challal Abdelmadjid Bouabdallah, Brahim Souici and Karima Benatchba "A New Weighted Shortest Path Tree for Convergecast Traffic Routing in WSN", (C2012 IEEE.

[18] Yankun Li, Ming Zhu and Haiqin Qu, Nannan Wang, Yichuan Song ," Improved Minimum Hop Routing 
Protocol in WSN", 2012 13th ACIS International Conference on Software Engineering, Artificial Intelligence, Networking and Parallel/Distributed Computing.

[19] Debashis De, Aditi Sen, Madhuparna Das Gupta," Cluster Based Energy Efficient Lifetime Improvement Mechanism for WSN with Multiple Mobile Sink and Single Static Sink" 2012 Third International Conference on Computer and Communication Technology.

[20] R. Valii and P . Dananjayan ," Power Control with QoS constraints in WSN using Game Theoretic Approach", IEEE-20180.

[21] Abderrazak Daoudi, Youssef Kerfi, Imade Benelallam, El Houssine Bouyakhf "A Constraint Programming approach for coverage optimization problem in WSN", 2012 IEEE.

[22] Ruqiang Yan, Senior Member, IEEE, Hanghang Sun, and Yuning Qian ," Energy-Aware Sensor Node Design With Its Application in Wireless Sensor Networks" , IEEE TRANSACTIONS ON INSTRUMENTATION AND MEASUREMENT, VOL. 62, NO. 5, MAY 2013.

[23] Ehsan Amiri1, Hassan Keshavarz2, Afshin Shahriari Fahleyani3, Hossein Moradzadeh4, and Shozo Komaki5
," New Algorithm for Leader Election in DistributedWSN with Software Agents", Proceeding of the 2013 IEEE International Conference on Space Science and Communication (IconSpace), 1-3 July 2013, Melaka, Malaysia.

[24] Monia Bellalouna, Afef Ghabri , "A Priori methods for Fault Tolerance in Wireless Sensor Networks.

[25] Tifenn Rault, Abdelmadjid Bouabdallah, Yacine Challal "WSN Lifetime Optimization through Controlled Sink Mobility and Packet Buffering", 2013 IEEE.

[26] Dilip Kumar," EECHE: Energy-Efficient Cluster Head Election Protocol for Heterogeneous Wireless Sensor Networks", International Conference on Advances in Computing, Communication and Control (ICAC3'09) ICAC3'09, January 23-24, 2009, Mumbai, Maharashtra, India. Copyright 2009 ACM 978-1-60558-351-8 (pp 7580)Year 2011.

[27] Alshahrany \& H. Zedan ,"A conceptual framework for Small WSN Configuration using Intelligent Decision Support System”, in 2013 